\title{
Infant and Young Child Feeding Practice Status and Associated Factors among Mothers of under 24-Month-Old Children in Shashemene Woreda, Oromia Region, Ethiopia
}

\author{
Fanos Yonas $^{1 *}$, Mekuria Asnakew ${ }^{2}$, Mekitie Wondafrash ${ }^{3}$, Misra Abdulahi $^{3}$ \\ ${ }^{1}$ Department of Public Health, Paradise Valley University College, Shashemene, Ethiopia \\ ${ }^{2}$ Department of Epidemiology, College of Public Health and medical Sciences, Jimma University, Jimma, \\ Ethiopia \\ ${ }^{3}$ Department of Population and Family Health, College of Public Health and Medical Sciences, Jimma University, \\ Jimma, Ethiopia \\ Email: fanosyonas@yahoo.com, Mekite.wondafrash@ju.edu.et, misra ab@yahoo.com, mekas63@yahoo.com
}

Received 5 June 2015; accepted 24 June 2015; published 1 July 2015

Copyright (C) 2015 by authors and OALib.

This work is licensed under the Creative Commons Attribution International License (CC BY). http://creativecommons.org/licenses/by/4.0/

(c) (i) Open Access

\section{Abstract}

Background: Infant and young child feeding is a cornerstone of care for childhood development. In Ethiopia, very large proportions of women do not practice optimal breastfeeding and complementary feeding for their children. Composite variables of infant and young child feeding index based on key indicators identified by WHO are not studied in Ethiopia; also there are no studies which document infant and young child feeding practices patterns and associated factors with inappropriate feeding practices in the study area. Objective: To assess infant and young child feeding practice (IYCFP) status and associated factors in Shashemene Woreda, Oromia Region. Methods: A community based cross sectional study was conducted from $01 / 04 / 2014$ to $30 / 04 / 2014$. A total of 423 mothers who had child less than two years were included in the study using simple random sampling technique. Data were collected using pre-tested semi structured interviewer administered questionnaire. Bi-variate and multivariate logistic regression models were used to identify factors associated with infant and young child feeding practices. Statistical significance was determined at $p$ value level less than 0.05 . Results: Overall, the prevalence of inappropriate infant and young child feeding practice in study area was $67.9 \%$. Being Government $[A O R=9.81(1.90$, $50.65)]$ and private $[A O R=7.66(1.40,41.94)]$ employee of husband, poorest socio-economic status $[A O R=1.39(1.30,6.48)]$, not attending ANC $(A O R=4.32(2.27,8.21)]$, child age 0 - 5 months $[A O R=16.01(5.01,50.03)]$, negative attitude of mothers $[A O R=2.50(1.14,5.47)]$ and number of

\footnotetext{
*Corresponding author.
}

How to cite this paper: Yonas, F., Asnakew, M., Wondafrash, M. and Abdulahi, M. (2015) Infant and Young Child Feeding Practice Status and Associated Factors among Mothers of under 24-Month-Old Children in Shashemene Woreda, Oromia Region, Ethiopia. Open Access Library Journal, 2: e1635. http://dx.doi.org/10.4236/oalib.1101635 
children 3 - 4 [AOR = $5.37(1.67,7.42)]$ were independent predictors of inappropriate IYCFP status. Conclusion: Inappropriate infant and young child feeding practice was prevalent in the study area. Interventional initiatives should focus on improving socio-economic status, and access to information, education and communication (IEC); also increasing ANC coverage for further improvement of IYCF is recommended.

\title{
Keywords
}

\author{
Infant and Young Child Feeding Practice, Ethiopia
}

Subject Area: Nutrition

\section{Introduction}

Infant and young child feeding is a cornerstone of care for childhood development. The first two years of life provides a critical window of opportunity for ensuring children's appropriate growth and development of children from generation to generation through optimal feeding. Any damage caused during this period can lead to impaired cognitive development, compromised educational achievement, low economic productivity and malnutrition because the first two years of a child's life is the most important for establishing healthy growth. Once growth faltering occurs in this age, there is little opportunity for catch-up growth. Stunted infants grow to be stunted children and stunted adults [1]-[3].

WHO and UNICEF's recommendations for optimal infant and young child feeding are early initiation of breastfeeding within one hour of birth, exclusive breastfeeding for six months and nutritionally adequate and safe complementary feeding starting from the age of six months with continued breastfeeding up to two years of age or beyond. The WHO also has developed indicators (eight core and nine optional) to assess infant and young child feeding practices [1]-[3].

Promotion of exclusive breastfeeding is the single most cost-effective intervention to reduce infant morbidity and mortality in developing countries. Even after complementary foods have been introduced at six month, breastfeeding remains a critical source of nutrients for the infant and young child. It provides about one half of an infant's energy needs up to the age of one year, and up to one third during the second year of life. Breast milk continues to supply higher quality nutrients than complementary foods, and also protective factors. Therefore, breastfeeding recommended up to 2 years and beyond with adequate complementary feeding [1]-[4].

Feeding practices for infant and young children worldwide are not optimal. It is only $34.8 \%$ of infants are exclusively breastfed worldwide. Complementary foods are often introduced too early or too late and are often nutritionally inadequate or unsafe [5]. Only about $39 \%$ of infants in the developing countries, $25 \%$ in Africa are exclusively breastfed for the first six months and $6 \%$ of infants in developing countries are never breastfed [6].

Globally, about $40 \%$ of under two years deaths are associated with inappropriate feeding practices [5]. Optimal breastfeeding and appropriate complementary feeding could prevent $13 \%$ and $6 \%$ under-five mortality respectively [7]. Over two third of malnutrition associated with inappropriate feeding practices during the first year of life [8].

Every day, 3000 - 4000 infants die in the developing world from diarrhoea and acute respiratory infections because they are given inadequate amounts of breast milk. More than 10 million children die each year in subSaharan Africa and South Asia. A major contributor to their deaths is poor breastfeeding practices [9]. The risk of death from diarrhoea of partially breastfed infants 0 - 6 months of age was 8.6 times the risk for exclusively breastfed children [10] [11].

A wide range of harmful infant and young child feeding practices were documented in Ethiopia. According to Ethiopia Demographic and Health Survey of 2011, 52\% of infants started breastfeeding within one hour of birth and exclusive breastfeeding during the first six months. About half (49\%) of children aged 6 - 8 months consumed solid, semi-solid, or soft foods and $5 \%$ of children were fed minimum dietary diversity and $4 \%$ of children fed minimum meal frequency per day while $96 \%$ of children continued breastfeeding at one year, and $82 \%$ continued at 2 year. Only $4 \%$ of children $6-23$ months living with their mothers are fed in accordance with IYCF practices and $66 \%$ children under the age of two receive age-appropriate breastfeeding. The prelacteal 
feeds within the first three days of life were $27 \%$, while $12 \%$ used a bottle with a nipple. In Oromia Region, $52.6 \%$ and $98.0 \%$ children started breast feeding within 1 hour and ever breast fed respectively [12].

Several efforts to improve the feeding status of the infant and young children have been carried out at different times. The Ethiopian government developed the Infant and Young Child Feeding (IYCF) guideline in 2004 following WHO recommendation of global strategy for feeding infants and young children for proper nutrition and health [13]. However, these efforts have failed to bring about substantive and sustainable changes leading to improvement of infant and young child feeding practices.

Composite variables of infant and young child feeding index based on key indicators identified by WHO are not studied in Ethiopia; also there are no studies which document infant and young child feeding practices patterns and associated factors with inappropriate feeding practices in the study area.

Accordingly, the objective of this study is to assess infant and young child feeding practice status and identify factors associated with it among mothers of children age less than 24 months in Shashemene Woreda.

\section{Methods and Materials}

\subsection{Study Design, Area and Study Population}

A community-based cross-sectional study design was employed. The study was conducted from 01/04/2014 to 30/04/2014. The study was carried out in Shashemene Woreda, West Arsi Zone, Oromia, Ethiopia, which is located at $250 \mathrm{~km}$ from Addis Ababa (capital city of Ethiopia). There are 13 Woredas in the Zone and total population is estimated to be $2,345,910$ according to 2013 projection. Shashemene Woreda total population and Households are 251,703 and 53,554 respectively. Among total population of Woreda, 14,372 are under two year children. There are 37 kebeles in Woreda. There are 6 Health Centers and 39 Health Post in Woreda. The source population was all mothers who had a child age less than 24 months and study population was mothers who had a child age less than 24 months in the randomly selected Kebele and who fulfills inclusion criteria. A single child per household who is in age group less than 24 month was included in the study. Those mothers who are unable to communicate due to serious illness at the time of data collection, known HIV positive status mothers and those children having any illness 15 days prior to the survey were excluded from the study because they affect feeding practices during data collection

\subsection{Sample Size Determination and Sampling Procedure}

The sample size was determined using single population proportion formula,

$$
n=\frac{\left(Z_{\left(\frac{\alpha}{2}\right)}\right)^{2} P(1-P)}{d^{2}}
$$

Considering the following assumption: prevalence of exclusive breast feeding 52\% [9], desired degree of precision of $5 \%$ and $\mathbf{Z} \alpha / \mathbf{2}$ is the value of the standard normal distribution corresponding to a significant level of alpha $(\alpha)$ of $\mathbf{0 . 0 5}$, which is $\mathbf{1 . 9 6}$. After adding $10 \%$ for non-response, the total sample size computed was 423 . According to the total number of study participant in each kebele, proportionate number of sample was assigned for each kebele. Study participants were selected by simple random sampling technique using random number computer generation method. Lists of children age less than 24 months that was prepared by Health Extension Works from family registration book was used as a sampling frame.

\subsection{Variables}

Dependent variable: Infant and young child feeding practice status Independent variables:

Socio demographic/economic characteristics: age of mother, occupation of mother, education status of mother, husband education status, husband occupation, husband substance use, family size;

$>$ Child characteristics: age, birth order, birth interval, perceived birth weight;

$>$ Maternal Health service factors: ANC, PNC, place of delivery, birth attendant, number of ANC visit, mode of delivery, IYCFP information during ANC, number of live births, number of children; 
Knowledge and Attitude of mothers or caregivers.

\subsection{Data Collection Method}

The data were collected using interviewer administered pre tested semi-structured questionnaire. Ten Public Health female students' from Paradise Valley University College as data collector and two supervisors (one Environmental Health and one BSC Nurse) were recruited.

\subsection{Data Processing and Analysis}

The data were edited, coded and entered in to EPI data version 3.1 and exported to SPSS version 16.0 statistical software for analysis. Further, data cleaning (editing, recoding, checking for missing values, and outliers) was made after exported to SPSS.

The data analysis ranges from the basic description to the identification of potential predictors of IYCFP. Bi-variate analysis and multivariable logistic models was used to show the relation between IYCFP and various associated factors.

Descriptive statistics were computed and presented using frequencies, proportions, summary statistics, graphs and tables.

Variables that have P-value $<0.25$ on bi-variate analyses were entered in the multivariate logistic regression model to identify independent predictors of IYCFP. All tests were two-sided and $\mathrm{P}<0.05$ was considered as statistically significant. The strength of association and precision were examined using adjusted odds ratio at $95 \%$ confidence interval.

\subsection{Measurements}

Meal frequency: measured based on data collected on solid, semi-solid, or soft foods within 24-hours dietary recall.

Dietary diversity: measured based on data collected on 7 foods groups within 24-hours dietary recall, which were grains, roots and tubers; legumes and nuts; dairy products (milk, yogurt, cheese); flesh foods (meat, fish, poultry and liver/organ meats); eggs; vitamin-A rich fruits and vegetables and other fruits and vegetables.

Wealth analysis: initially, reliability test was performed using the economic variables involved in measuring the wealth of the households. The variables which were employed to compute the alpha value were entered in to the principal component analysis. At the end of the principal component analysis, the wealth index was obtained as a continuous scale of relative wealth.

Age of the child: measured from the date of birth to the date of the survey. For those with written evidence, date of birth was obtained from child health cards and for those who didn't have written documents, age was established relying on the date given by the mother. To prevent recall bias of birth date local calendar with common local events was used.

\subsection{Data Quality Assurance}

The questionnaire was adapted from previous literatures \& modified in to the study context. It was prepared first in English and translated to Afan Oromo and then retranslated back to English by expert who is fluent in both languages to maintain its consistency..The questionnaire was also pre-tested and the finding from pretest was used to modify questionnaire and for formatting. Training was given to data collectors and supervisors. The principal investigator and supervisors checked completeness and clarity of each questionnaire on daily basis. They also checked the activities of each data collectors by random spot-checking of the households to ensure reliability of the data.

Any error, ambiguity, incompleteness, or any other problems were addressed on the following day before starting next day activities.

Probing questions were used to reduce recall bias in questions prone to error arising due to memory lapses.

\subsection{Ethical Consideration}

The study was conducted after obtaining ethical clearance from ethical review board of Jimma University. Letter of permission was secured from Woreda Health Office and Kebele administrators were communicated through 
formal letters from Woreda Health office in addition to personal communication.

Informed consent was obtained from each study subject prior to the interview after the purpose of the study is explained to respondent. Confidentiality of the information was assured and privacy of the respondent was maintained by removing personal identities.

\subsection{Operational Definitions}

Appropriate infant and young child feeding practice: defined as exclusive breast feeding in children age less than 6 months, early initiation of breast feeding, non use of bottle feeding, minimum meal frequency, minimum dietary diversity, timely introduction of solid, semi-solid and soft foods in 6 - 8 months and breast feeding. A practice that was appropriate for a specific age group received a score of 1 , and a practice that was inappropriate received a score of 0 .If summed score of the indicators is equal to 4 or above, it was considered as appropriate IYCFP [2].

Inappropriate infant and young child feeding practice: defined as non-exclusive breast feeding in children age less than 6 months, initiation of breast milk feeding after 1 hour, bottle feeding, food diversity less than 4 food groups, meal frequency less than three times per day, introduction of solid, semi-solid and soft foods before 6 months or after 8 months and non-breast feeding. If summed score is less than 4 score, it was considered as inappropriate IYCFP [2].

Pre lacteal feeding: children given something other than breast milk during the first three days of life.

Exclusive breast-feeding: infants, have received only breast-milk from his/her mother, and no other liquids or solids with the exception of drops or syrup consisting of vitamins, mineral or medicines. EBF measured for child less than 6 months only breast fed within 24 hour and if child is greater than 6 month by asking mother weather child fed only breast milk up to six month.

Predominant breast-feeding: the infants' predominant nourishment has been breast-milk and allows the infant to receive certain liquids (water and water-based drinks, fruit juice), ritual fluids and ORS, drops or syrups (vitamins, minerals, medicines).Non-human milk and food-based fluids are not allowed.

Continued breast feeding at 1 year: children $12-15$ months of age who are breast feed.

Continued breast feeding at 2 year: children $20-23$ months of age who are breast milk feed.

Complementary foods: any solid or semi-solid or soft foods which are given to the child.

Timely initiation of breast feeding: Putting neonate on mother breast to suck within 1 hour of delivery (including 1hour).

Ever breast feeding: breast feeding any time since birth.

Adequate (met) minimum dietary diversity: children $6-23$ months of age who consume 4 or more food groups from 7 food groups with 24 hours dietary recall.

Minimum meal frequency: children age 6 - 23 months who receive solid, semi-solid, or soft foods the minimum number ( 3 times for breastfed children and 4 times for non-breastfed children with 24 hours dietary recall.

Minimum acceptable diet: a composite indicator of minimum dietary diversity and minimum meal frequency. When a currently breastfed and non breast fed child meets both the minimum diversity and the minimum meal frequency.

Sufficient knowledge of IYCF: when the respondents correctly answer $60 \%$ or above $60 \%$ of questions about IYCF knowledge.

Insufficient knowledge of IYCF: when the respondents correctly answer below $60 \%$ of questions about IYCF knowledge.

Positive attitude about IYCF: when the respondents agree and strongly agree to favorable questions to appropriate IYCF.

Negative attitude about IYCF: When the respondents neutral, disagree and strongly disagree to favorable questions to appropriate IYCF.

Neutral: When mother's respond neither agrees nor disagree.

Family size: refers total number of people living in a house during the study period.

Young child: child less than 24 months.

Small BW: mothers' child size perception little baby during birth.

Normal BW: mothers' child size perception medium or normal during birth.

Large BW: mothers' child size perception big baby during birth. 
Substance use: use of any one substance such as drinking alcohol, chewing chat or smoking cigarette by husband sometimes/daily.

Wealth Index: is a composite measure of the cumulative living standard of a household.

\section{Results}

\subsection{Socio-Demographic Characteristics}

A total 417 of mothers and caregivers of children less than 24 months were included in the study yielding response rate of $98.6 \%$. The mean $( \pm \mathrm{SD})$ age of the mothers was 28.1 years $(\mathrm{SD} \pm 4.9)$ and ranges from 18 to 43 years. Majority of mothers $(91.6 \%)$ were married. More than half $(54.7 \%)$ were house wives by occupation. With regard to educational status, $170(40.7 \%)$ mothers did not have formal education. Most of respondents were Oromo by ethnicity $(88.2 \%)$ and Muslim by religion (86.2\%). Half $211(50.6 \%)$ of households had a family size four to six and the median family size was six. Regarding to wealth index one to five (20.6\%) of respondents were in $3^{\text {rd }}$ quartiles (Table 1 ).

With regards to husband education level, $53.7 \%$ of them had primary education while $230(60.2 \%)$ of husbands were farmers by occupation. Most (61.0\%) of husband didn't use substance (Table 1).

\subsection{Child Characteristics}

More than half (51.6\%) of children were males and more than one third (34.1\%) of them were 6-11 months old. More than two in five (47.7\%) of children were second to fourth in birth order. More than half of children (59.5\%) birth intervals between youngest child (index child) and his immediate older were less than 24 months. The majority (63.8\%) mothers perceived that their babies' birth weight was as normal (Table 2).

\subsection{Maternal Health Services Use Characteristics}

A total of 264 (63.3\%) mothers attended ante natal care. Among those who had ANC follow up, the majority $161(61.0 \%)$ had less than four visits with mean frequency of 3.22 times. During ANC follow up one third (76.5\%) of mothers didn't receive information about Infant and Young Child Feeding Practices. Majority (91.1\%) of mothers had spontaneous vaginal delivery. With respect to delivery assistance, 159 (38.1\%) mothers gave birth by the assistance of traditional birth attendants and $264(63.3 \%)$ gave birth at home. Most $(70.3 \%)$ of mothers didn't have PNC.

Most 369 (88.5\%) of mothers were multi-parous with mean of 4.2 live births and mean of total number of children they had was 3.96 (Table 3).

\subsection{Breast Feeding Practices}

Result of this study showed that almost all mothers 414 (99.3\%) had breast feed their children at least once (ever breast fed). Among those who ever breast fed children, 382 (92.3\%) were still breast feeding during the survey (currently on breast feeding). Among mothers who are currently breast feed and $382(92.3 \%)$ were also breast fed their children within 24 hour preceding the survey (yesterday). Among mothers who were not breast feeding their children currently, more than half (53.1\%) reported that they stopped breast feeding because it was time to stop (Table 4).

More than half of the mothers 246 (58.0\%) initiated breast-feeding within the 1st hour of delivery. A two third $(65.5 \%)$ of the mothers gave colostrum to their infants. The study also showed that $206(49.4 \%)$ mothers provided pre lacteal foods for baby and more than three quarter (79.1\%) of mothers didn't feed their children using bottle. Respondents were asked about time when they breast feed their child. They reported that they breast feed their child when child cries $331(86.6 \%)$. The frequency of breast feeding for those mothers who were currently breast feeding, $239(62.6 \%)$ of them reported that they were breastfeeding at least 8 times per day (Table 4).

Regarding exclusive breastfeeding, 79 (87.8\%) of infants in the age group 0 - 5 months were exclusively breastfed in the last 24 hours of the survey. During the survey mother with children age of 6 - 23 months were also asked retrospectively about exclusive breast feeding. One hundred forty nine (45.6\%) of mothers reported that they introduced foods and fluids before the child reach 6 month. Over all exclusive breast feeding for both age groups (0 - 5 months and 6 - 23 months) were 106 (38.4\%). 
Table 1. Socio-demographic characteristics of respondents in Shashemene Woreda, Oromia, Ethiopia from April 1 to April 302014 .

\begin{tabular}{|c|c|c|c|}
\hline \multicolumn{2}{|c|}{ Variables $(n=417)$} & \multirow{2}{*}{$\begin{array}{c}\text { Number } \\
8\end{array}$} & \multirow{2}{*}{$\begin{array}{c}\text { Percent } \\
1.9\end{array}$} \\
\hline \multirow{5}{*}{ Age (years) } & $\leq 19$ & & \\
\hline & $20-24$ & 81 & 19.4 \\
\hline & $25-29$ & 156 & 37.4 \\
\hline & $30-34$ & 124 & 29.7 \\
\hline & $\geq 35$ & 48 & 11.5 \\
\hline \multirow{4}{*}{ Marital status } & Married & 382 & 91.6 \\
\hline & Single & 13 & 3.1 \\
\hline & Widowed & 15 & 3.6 \\
\hline & Divorced & 7 & 1.7 \\
\hline \multirow{4}{*}{ Educational status } & No education & 170 & 40.8 \\
\hline & Primary education & 167 & 40.0 \\
\hline & Secondary education & 60 & 14.4 \\
\hline & Higher education & 20 & 4.8 \\
\hline \multirow{3}{*}{ Religion } & Muslim & 360 & 86.3 \\
\hline & Orthodox & 28 & 6.7 \\
\hline & Protestant & 29 & 7.0 \\
\hline \multirow{3}{*}{ Ethnicity } & Oromo & 368 & 88.2 \\
\hline & Amhara & 25 & 6.0 \\
\hline & Others & 24 & 5.8 \\
\hline \multirow{4}{*}{ Occupational status } & House wife & 228 & 54.7 \\
\hline & Farmer & 111 & 26.6 \\
\hline & Private & 62 & 14.9 \\
\hline & Government & 16 & 3.8 \\
\hline \multirow{3}{*}{ Family size } & $1-3$ & 46 & 11.0 \\
\hline & $4-6$ & 211 & 50.6 \\
\hline & $\geq 7$ & 160 & 38.4 \\
\hline \multirow{5}{*}{ Socio-economic status } & Poorest & 83 & 19.9 \\
\hline & Poorer & 81 & 19.4 \\
\hline & Middle & 86 & 20.6 \\
\hline & Richer & 84 & 20.1 \\
\hline & Richest & 83 & 19.9 \\
\hline \multirow{4}{*}{ Educational status of husband $(n=382)$} & No education & 38 & 9.9 \\
\hline & Primary education & 205 & 53.7 \\
\hline & Secondary education & 101 & 26.5 \\
\hline & Higher education & 38 & 9.9 \\
\hline \multirow{4}{*}{ Occupational status of husband $(\mathrm{n}=382)$} & Farmer & 230 & 60.2 \\
\hline & Merchant & 69 & 18.1 \\
\hline & Private & 52 & 13.6 \\
\hline & Government & 31 & 8.1 \\
\hline \multirow{2}{*}{ Husband substance use $(\mathrm{n}=382)$} & Yes & 149 & 39.0 \\
\hline & No & 233 & 61.0 \\
\hline
\end{tabular}


Table 2. Child characteristics in Shashemene Woreda, Oromia, Ethiopia from April 1 to April 302014.

\begin{tabular}{cccc}
\hline & Variable $(\mathbf{n}=\mathbf{4 1 7})$ & Number & Percent \\
\hline Sex & Male & 215 & 51.6 \\
& Female & 202 & 48.4 \\
& $0-5$ & 90 & 21.6 \\
Age (completed months) & $6-11$ & 142 & 34.1 \\
& $12-17$ & 125 & 14.4 \\
Birth order & $18-23$ & 60 & 11.8 \\
& First born & 49 & 47.7 \\
Preceding birth interval & $2^{\text {nd }}-4^{\text {th }}$ & 199 & 40.5 \\
( month) & $5^{\text {th }}$ or more & 169 & 11.8 \\
& No previous birth & 49 & 59.5 \\
Mother's perception of birth weight of child & Less than 24 & 248 & 28.7 \\
& More or equal to 24 & 120 & 11.0
\end{tabular}

Table 3. Maternal Health service characteristics in Shashemene Woreda, Oromia, Ethiopia from April 1 to April 302014.

\begin{tabular}{cccc}
\hline & Variable $(\mathbf{n}=\mathbf{4 1 7})$ & Number & Percent \\
\hline \multirow{2}{*}{ ANC visit } & No & 153 & 36.7 \\
& Less than four visit & 161 & 38.6 \\
& Four or more visit & 103 & 24.7 \\
Place delivery & Health Institutions & 153 & 36.7 \\
& Home & 264 & 63.3 \\
Mode of delivery & Vaginally & 380 & 8.1 \\
& Caesarean section & 37 & 33.6 \\
Birth attendant & Health professionals & 140 & 38.1 \\
& TBA & 159 & 28.3 \\
PNC Visit & Other & 118 & 29.7 \\
& Yes & 124 & 70.3
\end{tabular}


Table 4. Breast feeding practices among mothers of under 24 months in Shashemene Woreda, Oromia, Ethiopia from April 1 to April 302014.

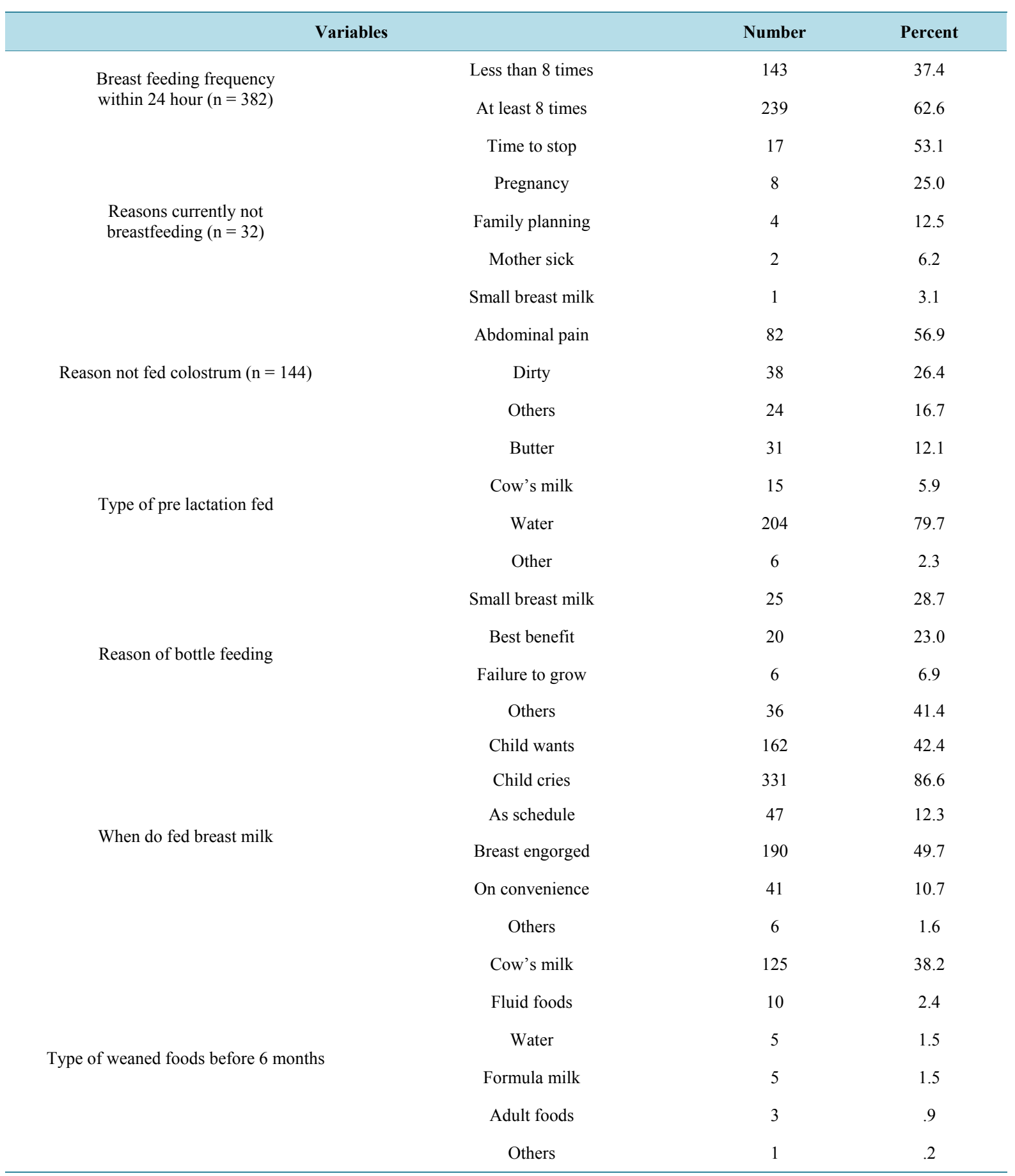

The proportion of children aged 6 - 23 months who were predominantly breastfed was 183 (56.0\%). Types of additional foods introduced before 6 month were, 125 (38.2\%) of them introduced cow's milk and 10 (2.4\%) fluid foods (Table 3).

Out of 106 mothers who had children age 12 - 15 months, almost all 103 (97.2\%) of mothers continued to breastfeed their children until the end of first year. However, among 48 children age 20 - 23 months, 29 (60.4\%) of them children continued to breast feed until the end of two years. 


\subsection{Complementary Feeding Practices}

The mean age for introduction of solid, semi-solid and soft foods was 5.6 $(\mathrm{SD} \pm 0.9)$ months. Among children age 6 - 23 months, $215(65.7 \%)$ started solid, semi-solid and soft foods when they were $6-8$ months age. Among children aged 6 - 23 months, only 128 (39.1\%) of children met the requirements for minimum dietary diversity. Two hundred sixty eight $(82.0 \%)$ of children met minimum meal frequency but $59(18 \%)$ of them consumed below minimum meal frequency requirement per day preceding 24 hour of survey. The proportion of children age 6 - 23 months who met minimum acceptable diet, composite of minimum dietary diversity and minimum meal frequency, was $111(33.9 \%)$ and $216(66.1 \%)$ didn't met requirement.

The primarily food source $(99.4 \%)$ of children aged $6-23$ months were grains, tubers and tubers whereas very smallest number $(4.3 \%)$ of children were provided with flesh products (Figure 1).

\subsection{Knowledge and Attitude of Mother's on IYCFP}

A total of sixty questions were included to assess the knowledge status about IYCFPs. Out of the total 417, 338 (81.1\%) of the respondents had sufficient knowledge on IYCFPs and $79(18.9 \%)$ of respondents had insufficient knowledge.

A total of eleven questions about IYCFP were asked to assess the attitude of respondents using Likert's scale. Out of 417 respondents $188(45.1 \%)$ had positive attitude and $229(54.9 \%)$ had negative attitude towards IYCFP.

\subsection{Infant and Young Child Feeding Practice Status}

Indicators used to construct comprehensive infant and young child feeding practices to classify into appropriate and inappropriate feeding were exclusively breast feeding, timely initiation of breast feeding, bottle feeding, timely introduction of solid, semi-solid and soft foods, minimum food diversification, minimum meal frequency and continued breast feeding.

Based on the above indicators of IYCFPs, $283(67.9 \%)$ of under 24 months children were fed inappropriately while 134 (32.1\%) were fed appropriately.

\subsection{Factors Associated with Infant and Young Child Feeding Practice Status}

Assessment of association between infant and young child feeding practices and socio-demographic status of

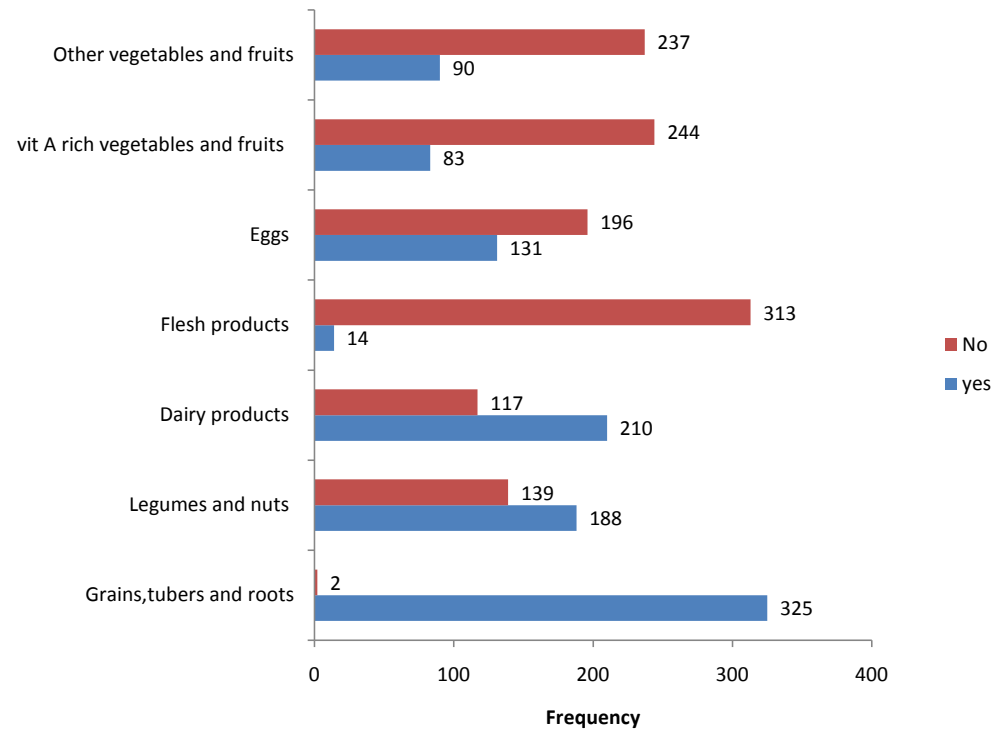

Figure 1. Types of food groups consumed within 24 hour prior to survey by children aged 6 - 23 months in Shashemene Woreda, Oromia, Ethiopia from April 1 to April 302014. 
respondent and husband, maternal health characteristics and child characteristics was made by employing binary logistic regression model.

In bi-variate analysis, occupation, mother educational status, husband occupation, husband educational status, family size, socio economic status, ANC visit, IYCF information, place of delivery, birth attendant, PNC, number of children, age of child, birth order and attitude of mothers toward IYCFP were significantly associated (P $<0.25$ ) and exported to multivariate logistic regression model to identify independent predictors of IYCFP and control confounding factors. However, marital status, husband substance use, mode of delivery, birth interval, perceived birth weight of child and knowledge of mothers on IYCFP were not significantly associated $(P>0.25)$ with infant and young child feeding practices in binary logistic regression and not selected for multivariate logistic regression model.

In multivariate analysis, adjusting possible confounding variables, husband occupation, socio economic status, ANC, number of children, age of child and attitude of mothers were significantly associated at $\mathrm{P}<0.05$ with infant and young child feeding practices. On the other hand, occupation of mothers, mother educational status, husband education status, family size, birth interval, birth order, birth attendant, IYCF information, place of delivery and PNC were not statistically associated $(\mathrm{P}>0.05)$ with IYCFP in this study (Table 5).

Mothers who were poorest 1.4 times more likely to practice inappropriate feeding when compared with richest $[\mathrm{AOR}=1.39(1.30,6.48)]$. The odds of inappropriate IYCPs are significantly higher for children their fathers were government and private employee than for merchant $[\mathrm{AOR}=9.81(1.90,50.65)$ and $\mathrm{AOR}=7.66(1.40$, 41.94)]. Infants in the age group $0-5$ months were 16 times more likely to practice inappropriate IYCF as compared to those children with age group 18 - 23 months [16.01 (5.01, 50.03)]. Mothers who didn't had ANC visit 4.3 times more likely to practice inappropriate IYCF when compared to those who had ANC visit [AOR $=4.32$ $(2.27,8.21)]$. Mothers who had negative attitude towards IYCFPs 2.5 times more likely to practice inappropriate IYCF when compared with those who have positive attitude $[\mathrm{AOR}=2.50(1.14,5.47)]$. Women with $3-4$ children 5.4 times more likely practice inappropriate IYCF compared with women with children $1-2$ [AOR = $5.37,(1.65,7.42)]$.

\section{Discussion}

The aim of this study was to assess infant and young child feeding practice status and associated factors in Shashemene Woreda, Oromia Region. In this study, it was found that majority $(99.3 \%)$ of mothers practiced ever breastfeeding. This result is nearly similar with the study done in Mekelle town in 2011 which was 98.9\% [14] and Ethiopian ever breast feeding rate was 98\% in 2011 [12]. But it is higher than the ever breastfeeding in Arbaminch which was $95.8 \%$ in 2011 [15]. This high rate of breastfeeding in study is may be due to the fact that breast feeding practice is a common, accessible and norm in the society.

Although WHO's, Global and National Infant, and Young Child Feeding Guidelines recommend that all newborns should start breastfeeding immediately (within the first hour after delivery), this study shows that $58.0 \%$ mothers started to breastfeed immediately within one hour after birth. This finding is lower than study done in Mekelle (78\%) in 2011 [14] and nearly similar to Ethiopia (52\%) in 2011 [12]. This study finding is below HSDP IV targets (80\%) [13]. This low proportion of initiation of breast feeding within 1 hour of delivery according to recommendation of WHO may be due to socio-cultural constraints or may be due to the mothers don't know when to initiate breast feeding after delivery.

Although Global strategy on infant and young child feeding recommends feeding colostrum and discourages pre-lacteal feeds, in this study $65.5 \%$ of mothers gave colostrums to their baby. This finding was lower than the finding of Mekelle town (82\%) in 2011 [14] and Arbamincharea (89.8\%) in 2011 [15] and higher than study done in Dabat town 51.9\% in 2006 [16]. This result may be justified that in the study area mothers discarded colostrum as they considered that it cause of diseases and abdominal pain (56.9\%), dirty (26.4\%) and it is water $(16.7 \%)$

The prevalence of pre-lacteal feeding in this study is $49.4 \%$ which is much higher than the study done in Mekelle town (10.4\%) in 2011 [14], in south Gonder zone (11.1\%) in 2007 [17] and 27\% in EDHS 2011 [12]. This result may be due to the tradition ("wubaxe") of introducing pre-lacteal feeding especially water $(79.7 \%)$ in third day of birth and high home delivery (63.3\%) in the study.

This study showed that the prevalence of exclusive breastfeeding for infants less than six months was $87.8 \%$. This result is better when compared with the findings from Mekelle 60.8\% in 2011 [14] and Ethiopia (52\%) in EDHS 2011 [12]. This finding is also higher than the national targets (70\%) by 2015 [13]. The reason for this high 
Table 5. Multivariable logistic regression models predicting IYCFP status, Shashemene Woreda, Oromia, Ethiopia from April 1 to April 302014.

\begin{tabular}{|c|c|c|c|c|c|}
\hline \multicolumn{6}{|c|}{ IYCFP status } \\
\hline \multicolumn{2}{|c|}{ Variables } & \multicolumn{3}{|c|}{ COR $(95 \%$ C.I $)$} & \multirow[t]{2}{*}{ AOR (95\% C.I) } \\
\hline & & Appropriate (\%) & In & ate $(\%)$ & \\
\hline \multirow{4}{*}{$\begin{array}{l}\text { Education status of } \\
\text { mothers }\end{array}$} & No & $30(17.6)$ & $140(82.4)$ & $8.67(3.19,23.56)^{*}$ & $2.63(0.23,29.59)$ \\
\hline & Primary & $60(35.9)$ & $107(64.1)$ & $3.31(1.25,8.75)^{*}$ & $1.85(0.19,1814)$ \\
\hline & Secondary & $31(51.7)$ & $29(48.3)$ & $1.74(0.60,4.96)$ & $2.59(0.30,22.17)$ \\
\hline & Higher & $13(65)$ & $7(35)$ & 1.00 & 1.00 \\
\hline \multirow{4}{*}{$\begin{array}{l}\text { Occupational status } \\
\text { of mother }\end{array}$} & Government & $11(68.8)$ & $5(31.2)$ & 1.00 & 1.00 \\
\hline & Private & $35(56.5)$ & $27(43.5)$ & $1.70(0.53,5.47)$ & $0.51(0.05,5.40)$ \\
\hline & Farmer & $30(27)$ & $81(73)$ & $5.94(1.91,18.52)^{*}$ & $0.25(0.02,3.10)$ \\
\hline & Housewife & $58(25.4)$ & $170(74.6)$ & $6.45(2.15,19.34)^{*}$ & $0.44(0.04,5.14)$ \\
\hline \multirow{4}{*}{$\begin{array}{c}\text { Educational status } \\
\text { of husband }\end{array}$} & No & $6(15.8)$ & $32(84.2)$ & $10.26(3.42,30.80)^{*}$ & $0.81(0.09,7.50)$ \\
\hline & Primary & $45(22)$ & $160(78)$ & $6.84(3.24,14.44)^{*}$ & $1.56(0.32,7.62)$ \\
\hline & Secondary & $42(41.6)$ & $59(58.4)$ & $2.70(1.24,5.88)^{*}$ & $0.90(0.22,3.70)$ \\
\hline & Higher & $25(65.8)$ & $13(34.2)$ & 1.00 & 1.00 \\
\hline \multirow{4}{*}{$\begin{array}{c}\text { Occupational status } \\
\text { of husband }\end{array}$} & Government & $23(74.2)$ & $8(25.8)$ & $0.13(0.05,0.35)^{*}$ & $9.81(1.90,50.65)^{*}$ \\
\hline & Private & $15(28.8)$ & $37(71.2)$ & $0.94(0.42,2.09)$ & $7.66(1.40,41.94)^{*}$ \\
\hline & Farmer & $61(26.5)$ & $169(73.5)$ & $1.05(0.58,1.92)$ & $4.52(0.69,29.55)$ \\
\hline & Merchant & $19(27.5)$ & $50(72.50$ & 1.00 & 1.00 \\
\hline \multirow{3}{*}{ Family size } & $1-3$ & $29(63)$ & $17(37)$ & 1.00 & 1.00 \\
\hline & $4-6$ & $72(34.1)$ & $139(65.9)$ & $0.15(0.75,0.31)^{*}$ & $0.38(0.02,7.35)$ \\
\hline & $=>7$ & $33(20.6)$ & $127(79.4)$ & $0.50(0.31,0.81)^{*}$ & $0.58(0.02,17.07)$ \\
\hline \multirow{5}{*}{$\begin{array}{c}\text { Socio-economic } \\
\text { status }\end{array}$} & Poorest & $45(54.2)$ & $38(45.8)$ & $0.31(0.16,0.58)^{*}$ & $1.39(1.30,6.48)^{*}$ \\
\hline & Poorer & $25(30.9)$ & $56(69.1)$ & $0.81(0.41,1.59)$ & $2.71(0.66,11.07)$ \\
\hline & Middle & $27(31.4)$ & $59(68.6)$ & $0.79(0.40,1.54)$ & $2.18(0.53,9.01)$ \\
\hline & Richer & 15 (17.9) & $69(82.1)$ & $1.65(0.79,3.48)$ & $2.57(0.78,8.48)$ \\
\hline & Richest & $22(26.5)$ & $61(73.5)$ & 1.00 & 1.00 \\
\hline \multirow{4}{*}{$\begin{array}{l}\text { Age of child } \\
\text { (month) }\end{array}$} & $0-5$ & $4(4.4)$ & $86(95.6)$ & $15.36(4.98,47.36)^{*}$ & $16.01(5.01,51.03)^{*}$ \\
\hline & $6-11$ & $43(30.3)$ & $99(69.7)$ & $1.65(0.88,3.08)$ & $2.00(0.67,5.96)$ \\
\hline & $12-17$ & $62(49.6)$ & $63(50.4)$ & $0.73(0.39,1.35)$ & $0.76(0.24,2.40)$ \\
\hline & $18-23$ & $25(41.7)$ & $35(58.3)$ & 1.00 & 1.00 \\
\hline \multirow{3}{*}{ Birth order } & $1^{\text {st }}$ & $31(63.3)$ & $18(36.7)$ & $0.13(0.07,0.26)^{*}$ & $0.18(0.00,8.09)$ \\
\hline & $2-4$ & $72(36.2)$ & $127(63.8)$ & $0.40(0.24,0.64)^{*}$ & $0.54(0.05,6.43)$ \\
\hline & $=>5$ & $31(18.3)$ & $138(81.7)$ & 1.00 & 1.00 \\
\hline
\end{tabular}




\begin{tabular}{|c|c|c|c|c|c|}
\hline \multicolumn{6}{|l|}{ Continued } \\
\hline \multirow{3}{*}{ No. of children } & $1-2$ & $58(56.3)$ & $45(43.7)$ & 1.00 & 1.00 \\
\hline & $3-4$ & $46(28.6)$ & $115(71.4)$ & $3.22(1.92,5.41)^{*}$ & $5.37(1.65,17.42)^{*}$ \\
\hline & $>=5$ & $30(19.6)$ & $123(80.4)$ & $5.28(3.02,9.23)^{*}$ & $2.99(0.12,74.71)$ \\
\hline \multirow{2}{*}{ ANC } & Yes & $113(42.8)$ & $151(57.2)$ & 1.00 & 1.00 \\
\hline & No & $21(13.7)$ & $132(86.3)$ & $4.70(2.79,7.92)^{*}$ & $4.32(2.27,8.21)^{*}$ \\
\hline \multirow{3}{*}{ Place of delivery } & HI & $68(44.4)$ & $85(55.6)$ & 1.00 & 1.00 \\
\hline & Home & $66(25)$ & $198(75)$ & $2.40(1.57,3.67)^{*}$ & $4.35(0.65,28.96)$ \\
\hline & HP & $63(45)$ & $77(55)$ & 1.00 & 1.00 \\
\hline \multirow[t]{2}{*}{ Birth attendant } & TBA & $40(25.2)$ & $119(74.8)$ & $2.43(1.49,3.97)^{*}$ & $0.24(0.03,1.92)$ \\
\hline & Others & $31(26.3)$ & $87(73.7)$ & $2.30(1.35,3.89)^{*}$ & $0.29(0.04,2.11)$ \\
\hline \multirow{2}{*}{$\mathrm{PNC}$} & Yes & $49(39.5)$ & $75(60.5)$ & 1.00 & 1.00 \\
\hline & No & $85(29)$ & $208(71)$ & $1.60(1.03,2.48)^{*}$ & $0.76(0.34,1.72)$ \\
\hline \multirow{2}{*}{ IYCF information } & Yes & $96(47.5)$ & $106(52.5)$ & 1.00 & 1.00 \\
\hline & No & $17(27.4)$ & $45(72.6)$ & $2.40(1.29,4.47)^{*}$ & $1.79(0.71,4.51)$ \\
\hline \multirow{2}{*}{ Attitude of mothers } & Negative & $61(26.6)$ & $168(73.4)$ & $1.75(1.16,2.65)^{*}$ & $2.50(1.14,5.47)^{*}$ \\
\hline & Positive & $73(38.8)$ & $115(61.2)$ & 1.00 & 1.00 \\
\hline
\end{tabular}

${ }^{*} \mathrm{P}$ value $<0.05$.

prevalence of exclusive breast feeding in study area might be the result of the current policy implementation on the use of health extension workers in rural areas to promote breastfeeding. The methods used to calculate exclusive breast feeding for 0 - 5 months children are only 24 hour recall of exclusiveness. This may contribute for high proportion of exclusive breast feeding. This way of determining of exclusive breast feeding may not show absolutely weather the child is on exclusive breast feeding.

Using a bottle with a nipple for feeding infant, a practice that is discouraged increases the child's risk of illness and reduces the child's interest in breastfeeding, with consequent potential decline in milk production. The prevalence of bottle feeding in this study was 20.9\% which is higher than EDHS finding (12\%) in 2011 [12] and $10 \%$ in Western Uganda [18] and Tanzania 2010 [19]. This is may be due to many mothers in the study area perceived that bottle feeding has more benefit than breast feeding $(23.8 \%)$ and increases growth of children $(6.9 \%)$.

Introduction of nutritionally adequate and safe complementary foods promotes growth and good nutritional status among infants and young children. This study revealed that, $65.7 \%$ of children aged $6-8$ months receive complementary foods. The finding of this study is higher when compared with EDHS (49\%) in 2011 [12]. This is low when compared with Uganda (75\%) [18], Tanzania (92.3\%) [19] and Kenya (81\%) [7] in 2010.This is may be due to mothers' knowledge on what and when to start additional foods to child and their perception that the child is unable to digest foods in this age.

This study also showed that the majority $82.0 \%$ of children received minimum meal frequency. However, very few $39.1 \%$ children aged $6-23$ months met the requirements for minimum dietary diversity in the previous day. This is higher than in Tanzania, $38.0 \%$ of children age received minimum dietary diversity and $35 \%$ of children ages 6 - 23 months meet minimum meal frequency in 2010 [19]. In Western Uganda 49\% of 6 - 23 months children complemented 3 or 4 times [18]. Similarly in South Asia, the minimum meal frequency and dietary diversity was reported by WHO in 2010 to be less than $50 \%$ in all countries except Sri Lanka [20]. The study finding also high when compared to EDHS 2011 (minimum meal frequency $4 \%$ and minimum dietary diversity (5\%) [12]. This is may be due to difference used to calculate minimum meal frequency. In this study the proportion of minimum meal frequency was calculated for $6-23$ months without considering age specific meal frequency i.e. 6 - 8 months, 9 - 11 months and 12 - 23 months.

In this study, $99.4 \%$ of food groups given to children are mainly made from grains, roots and tubers, which have relatively low nutrient density. This study also found that, meat products (4.3\%), eggs (40.1\%) and vitamin A rich foods (25.4\%). This is similar with India, consumption of animal origins foods was found to be poor especially for younger age group and less than $8 \%$ of all children aged $6-23$ months had consumed eggs, meat, 
poultry and fish during the past 24 hours [21]. In Tanzania 2010, Proportion of children who were given food made of grains, roots and tubers (93\%) and vitamin A rich fruits and vegetables $(67.2 \%)$ and animal origin food was very low [19]. According to EDHS 2011, foods made from grains (66\%) are consumed more often than foods from any other food group (only $15 \%$ consumed fruits and vegetables rich in vitamin A, $5 \%$ of children consumed meat and $8 \%$ consumed eggs) [12]. This is may be due to negative beliefs of mothers or caregivers or lack of affordability of these foods or inadequate knowledge about the importance of feeding young children variety of foods among mothers and caregivers or associated with household food security.

In the binary logistic regression model, mother occupation, mother educational status, husband occupation, husband educational status, family size, socio economic status, ANC visit, IYCF information, place of delivery, birth attendant, PNC, number of children, age of child, birth order and attitude of mothers toward IYCFP were significantly associated with IYCFPs $(\mathrm{P}<0.25)$.

However, inappropriate infant and young child feeding practice was significantly associated with husband occupation, socio economic status, ANC, number of children, age of child and attitude of mothers after controlling other predictors in the multiple logistic regression model.

Seven infant and young child feeding practices indicators were assessed based on WHO recommendation and used to develop composite variable. Identification of factors affecting IYCFP is important as it can guide implementation of appropriate programs to improve IYCF practices. In this study it was found that only $32.1 \%$ of children received appropriate feeding practice. The WHO guidelines on infant and young child feeding practices do not provide the baseline or the minimum standard that needs to be reached nor what percentage should be considered alarming for public health significance. Logically, it is desirable that all children 0 - 23 months meet the recommended feeding practices.

To appreciate similarities and differences of factors associated with inappropriate infant and young child feeding practice in logistic regression with other studies are impossible due to lack of study on IYCFP in composite of similar indicators.

The differences in the measurement of the feeding practices, and the real meaning of each practice could be sources of difficulty for building and interpreting composite feeding practice, and perhaps this is also a source of confusion for defining its indices. Despite the lack of a standard definition and the variations in the methods used to construct scores.

The findings of this study should be interpreted with some limitations. As a result of cross-sectional study design, the temporal sequence of events cannot be determined. Recall bias and social desirability bias are also potential limitations that may have been encountered in this study.

\section{Conclusions and Recommendation}

In conclusion, inappropriate IYCFP was prevalent in the study area. This prevalence is not acceptable to ensure good health and better nutritional status of children and to achieve 4th MDGs. The study showed high proportion of mothers giving pre-lacteal fed, discarding colostrums and using nipple bottle for feeding.

Being government, poorest socio-economical status, not attending ANC visit child age 0 - 5 months, negative attitude of mothers and number of children $(3-4)$ were significantly associated with infant and young child feeding practice at $\mathrm{P}<0.05$.

Further, interventional initiatives should focus on improving socio-economic status. Attitudes affect IYCFPs and ANC utilization for further improvement of IYCFP. In addition, longitudinal studies also needed to carefully track IYCFP from birth to 24 months of age and causally link these practices with individual child.

\section{Authors' Contributions}

Fanos Y., Mekite W., Misra A. and Mekuria A. participated in the conception, design of the study, analysis and interpretation of the data.

Fanos Y. and Mekuria A. drafted the manuscript and coordinated the data collection. Mekite W. and Misra A. involved in the review of the manuscript. All authors read and approved the final manuscript.

\section{Competing Interests}

We declare that we do not have competing interests. 


\section{Acknowledgements}

We gratefully acknowledge the study participants, supervisors and data collectors for the information provided, without which this work would not have been possible. The funders had no role in the study.

\section{References}

[1] WHO/UNICEF (2003) Global Strategy for Infant and Young Child Feeding. World Health Organization, Geneva.

[2] WHO/UNICEF/USAID/FANTA/IFPRI (2008) Indicators for Assessing Infant and Young Child Feeding Practices: Part 1: Definitions. World Health Organization, Geneva.

[3] WHO/UNICEF/USAID/FANTA/IFPRI (2010) Indicators for Assessing Infant and Young Child Feeding Practices: Part 2: Measurement. World Health Organization, Geneva.

[4] Kramer, M.S. and Kakuma, R. (2001) The Optimal Duration of Exclusive Breastfeeding: A Systematic Review. World Health Organization, Geneva.

[5] PAHO/WHO (2002) Guiding Principles for Complementary Feeding of the Breastfed Child. Pan American Health Organization/World Health Organization, Washington DC.

[6] WHO/UNICEF (2006) Infant and Young Child Feeding Counseling: An Integrated Course. Participant's Manual.

[7] Kimani-Murage, E., et al. (2011) Patterns and Determinants of Breastfeeding and Complementary Feeding Practices in Urban Informal Settlements, Nairobi Kenya. BMC Public Health, 11, 396. http://www.biomedcentral.com/1471-2458/11/396

[8] Ministry of Human Resource Development, Department of Women and Child Development, Food and Nutrition Board (2004) National Guidelines Infant and Young Child Feeding Practices. Government of India, New Delhi.

[9] Kibebew, A. (2012) Infant and Young Child Feeding Practices among mothers Living Harar Town. Harar Bulletin of Health Sciences, Extracts 4, 66-78

[10] Jones, G. (2003) How Many Child Deaths Can We Prevent This Year? The Lancet, 362, 65-71. http://dx.doi.org/10.1016/S0140-6736(03)13811-1

[11] Bahl, R., Frost, C., Kirkwood, B.R., Edmond, K., Martines, J., Bhandari, N. and Arthur, P. (2005) Infant Feeding Patterns and Risks of Death and Hospitalization in the First Half of Infancy: Multicentre Cohort Study. Bulletin of the World Health Organization, 83, 418-426.

[12] CSA of Ethiopia and ICF International (2012) Ethiopia Demographic \& Health Survey of 2011. Addis Ababa and Calverton, MD.

[13] Family Health Department (2004) National Strategy for Infant \& Young Child Feeding Federal Ministry of Health. Addis Ababa.

[14] Berhe, H., Mekonnen, B., Bayray, A. and Berhe, H. (2011) Determinants of Breast Feeding Practices among Mothers Attending Public Health Facilities, Mekelle, Northern Ethiopia: A Cross Sectional Study. International Journal of Pharmaceutical Sciences and Research, 4, 650-660.

[15] Tamiru, D., Bogale, B. and Merdekios, B. (2013) Breastfeeding Patterns and Factors Associated with Exposure to SubOptimal Breastfeeding Practices in Rural Communities of Arba Minch Zuria, Ethiopia. Global Health Perspectives, 1, $105-112$

[16] EPHA, Abstract 11, Assessment of Infant and Young Child Feeding Practice in Dabat Town, North West Ethiopia, 2006.

[17] Nekatebeb, H., Guyon, A., Beyero, M. and Stoecker, B.J. (2010) Factors Related to Exclusive Breast Feeding and Dietary Diversity of Complementary Foods: A Case Study in Amhara Region of Ethiopia. Master's Thesis, Addis Ababa University, Addis Ababa.

[18] Wamani, H., Peterson, S., Tylleskar, T. and Tumwine, J.K. (2005) Infant and Young Child Feeding in Western Uganda: Knowledge, Practices and Socio-Economic Correlates. Journal of Tropical Pediatrics, 51, 356-361. http://dx.doi.org/10.1093/tropej/fmi048

[19] Victor, R. (2012) Infant and Young Child Feeding Practices among Children Aged 0 - 23 Months in Tanzania. Maternal and Child Nutrition Journal, 10, 1740-1720-1929. http://hdl.handle.net/1959.13/939120

[20] Pandey, S., Tiwari, K., Senarath, U., Agho, K.E. and Dibley, M.J., South Asia Infant Feeding Research Network (2010) Determinants of Infant and Young Child Feeding Practices in Nepal: Secondary Data Analysis of Demographic and Health Survey 2006. Food and Nutrition Bulletin, 31, 334-351.

[21] Sinhababu, A. (2010) Infant and Yong Child Feeding Practices in Bankura District, West Bengal, India. Journal of Health, Population and Nutrition, 28, 294-299. 